\title{
The expression of NeuroD and mASH1 in the gastroenteropancreatic neuroendocrine tumors
}

\author{
Takashi Shida ${ }^{1,2}$, Mitsuko Furuya ${ }^{2}$, Takashi Kishimoto², Takashi Nikaido ${ }^{3}$, \\ Tohru Tanizawa $^{6}$, Keiji Koda ${ }^{4}$, Kenji Oda ${ }^{5}$, Shigetsugu Takano ${ }^{1}$, Fumio Kimura ${ }^{1}$, \\ Hiroaki Shimizu $^{1}$, Hiroyuki Yoshidome ${ }^{1}$, Masayuki Ohtsuka ${ }^{1}$, Yukio Nakatani ${ }^{6}$ \\ and Masaru Miyazaki ${ }^{1}$
}

${ }^{1}$ Department of General Surgery, Chiba University Graduate School of Medicine, Chiba, Japan; ${ }^{2}$ Department of Molecular Pathology, Chiba University Graduate School of Medicine, Chiba, Japan; ${ }^{3}$ Department of Pathology, Jikei University School of Medicine, Tokyo, Japan; ${ }^{4}$ Department of Surgery, Teikyo University, Chiba, Japan; ${ }^{5}$ Department of Surgery, Chiba Aoba Hospital, Chiba, Japan and ${ }^{6}$ Department of Diagnostic Pathology, Chiba University Graduate School of Medicine, Chiba, Japan

\begin{abstract}
Gastroenteropancreatic neuroendocrine tumors are uncommon and their tumor biology has not been well elucidated to date. Currently the WHO classification is widely used for the diagnosis and distinction of this tumor entity, which is sometimes cumbersome. Although neuroendocrine tumor markers do exist (ie chromograninA, synaptopyhsin, etc), sensitive and specific markers that accurately predict tumor growth and tumor behavior are still absent. In the present study, we assessed the expression of transcription factors (NeuroD and mASH1) essential for the normal fetal neuronal development in 33 gastroenteropancreatic neuroendocrine tumor patients (12 well-differentiated neuroendocrine tumors, 7 well-differentiated neuroendocrine carcinomas, and 14 poorly differentiated neuroendocrine carcinomas). NeuroD was less expressed in poorly differentiated neuroendocrine carcinoma (small-cell type) compared to well-differentiated neuroendocrine tumor (carcinoid) by reverse transcription-polymerase chain reaction. Immunohistochemical staining revealed that mASH1 was highly (sensitivity of $71 \%$ ) and specifically (specificity of $95 \%$ ) expressed in poorly differentiated neuroendocrine carcinoma. High NeuroD expression was seen in all well-differentiated neuroendocrine carcinoma and tumor (carcinoid) patients. Low NeuroD expression was seen in $36 \%$ (5 of 14) of poorly differentiated neuroendocrine carcinoma patients, which was associated with significant shorter overall survival. The expression pattern of these transcription factors may represent the biological and pathophysiological difference of gastroenteropancreatic neuroendocrine tumors and may become a new marker for the distinction of gastroenteropancreatic neuroendocrine tumors.
\end{abstract}

Modern Pathology (2008) 21, 1363-1370; doi:10.1038/modpathol.2008.121; published online 27 June 2008

Keywords: NeuroD; mASH1; hASH1; gastroenteropancreatic; neuroendocrine tumor

Neuroendocrine tumors are a group of tumors originating from cells of the diffuse endocrine system. The diffuse endocrine system includes a number of endocrine cells spreading throughout all the body with the ability to secrete bioactive peptides and amines. Gastroenteropancreatic neuroendocrine tumors are fairly rare, and the pathophysiology of this tumor entity has not been well

Correspondence: Dr T Shida, MD, PhD, Department of General Surgery, Chiba University Graduate School of Medicine, 1-8-1 Inohana, Chuo-ku, Chiba 260-8670, Japan.

E-mail: shidax812@yahoo.co.jp

Received 24 April 2008; revised 27 May 2008; accepted 28 May 2008; published online 27 June 2008 documented to date. Moreover diagnosis and target treatment is limited and management is challenging because little is known about the cell biology and mechanistic regulation of these tumors. ${ }^{1}$ Gastroenteropancreatic neuroendocrine tumors are classified according to the WHO classification into welldifferentiated tumors and carcinomas or poorly differentiated carcinomas. ${ }^{2}$ The current WHO classification system uses both stage-related criteria (size and presence of metastases) and grade-related criteria (mitotic rate, perineural invasion, angioinvasion, and Ki-67 proliferative index) for classifying and predicting the outcome of gastroenteropancreatic neuroendocrine tumors. As this approach includes the majority of well-accepted pathologic 
prognostic factors, it has been presumed to be more accurate in predicting the outcome, but its clinical application is cumbersome. The multiple grading parameters may make it difficult to reliably reproduce this system between pathologists and institutions.

Achaete-scute homologue 1 (termed mASH1 in rodents, hASH1 in humans) is a basic helixloop-helix (bHLH) transcription factor that is essential in the early development of neural and neuroendocrine progenitor cells in many tissues. ${ }^{3}$ hASH1(mASH1) has been reported to be abundantly expressed in gastrointestinal neuroendocrine carcinomas as well as in pulmonary small-cell carcinoma., ${ }^{4,5}$ Another member of the bHLH transcription factor, NeuroD is expressed transiently in developing neurons in the central and peripheral nervous systems but is not expressed in nearby neuronal progenitors that are still undergoing cell division. ${ }^{6}$ NeuroD has been reported to be expressed in pituitary adenomas, pulmonary large-cell neuroendocrine carcinomas, and carcinoids and also in some gastric carcinomas with neuroendocrine feature but not in small-cell carcinomas. ${ }^{7-9}$ The expression of these transcription factors has not been fully elucidated in gastroenteropancreatic neuroendocrine tumors.

The aim of this study was to determine the expression patterns of these neuronal transcription factors in gastroenteropancreatic neuroendocrine tumors (both neuroendocrine carcinomas and carcinoids) and its relation to clinicopathological, phenotypical characteristics including their prognosis. Moreover to seek whether these neuronal transcription factors serve as a new marker for gastroenteropancreatic neuroendocrine tumors, although neuroendocrine tumor markers do exist (ie chromograninA, synaptophysin), sensitive and specific markers that predict tumor growth and behavior are absent.

\section{Materials and methods}

\section{Patients and Tissues}

Formalin-fixed and paraffin-embedded samples of gastroenteropancreatic neuroendocrine tumors $(n=33)$ were obtained from Department of General Surgery, Chiba University Hospital, Japan, from 1999 to 2007. The patient age ranged from 19 to 83 years. Patients who underwent neoadjuvant therapy, and with carcinomas besides gastroenteropancreatic neuroendocrine tumors were excluded. Written informed consent was obtained from each patient. Clinical and pathological data were documented and entered into a specific tumor registry after surgery (or endoscopic mucosal resection) and follow-up (Table 1). In two of the cases (case no. 2 and 4), surgery was not performed because of extensive tumor spread, and biopsy specimens were used for the study.
Pathologic diagnosis of gastroenteropancreatic neuroendocrine tumors was done based on the WHO classification. ${ }^{2}$ All tumors were categorized into three groups: well-differentiated neuroendocrine tumor (carcinoid, $n=12$ ), well-differentiated neuroendocrine carcinoma $(n=7)$, and poorly differentiated neuroendocrine carcinoma $(n=14)$. Furthermore, poorly differentiated neuroendocrine carcinomas were subdivided into small-cell type $(n=8)$ and large-cell type $(n=6)$ on the basis of histological findings using the criteria commonly applied to pulmonary neuroendocrine carcinomas. ${ }^{9}$ The histological feature of small-cell neuroendocrine carcinomas was minimal cytoplasm, a fusiform cell shape, finely granular chromatin, small or absent nucleoli, and nuclear molding. The histological feature of large-cell neuroendocrine carcinomas was more cytoplasm, a round or polygonal cell shape, prominent nucleoli, and a coarse chromatin pattern.

\section{Immunohistochemistry}

All tumors were examined by immunohistochemical staining. Sections (4 um) were cut from formalin-fixed paraffin-embedded tissues and placed on silanized slides. The antibodies used were as follows: polyclonal chromograninA antibody (ready to use; Nichirei, Tokyo, Japan), polyclonal synaptophysin antibody (ready to use; DakoCytomation), polyclonal Neuro D antibody (1:200; N-19; Santa Cruz Biotechnology, Santa Cruz, CA, USA), monoclonal mASH1 antibody (1:50; 24B72D11.1; Becton Dickinson Biosciences, Franklin Lakes, NJ, USA), and monoclonal MIB-1 antibody (1:100; DakoCytomation). Staining was done by using labeled streptavidin-biotin-peroxidase and autoclave antigen retrieval technique. The staining pattern was scored as follows: - , no staining or $<5 \%$ of the tumor cells positive; + , occasional weak staining; ++ , moderate staining; and +++ , intense staining. To obtain the percentage of Ki-67 (MIB-1) immunoreactive nuclei in each tumor, three random fields at $\times 200$ magnification were taken. The Ki-67 (\%) was determined and expressed as the percentage of cells with Ki-67 immunoreactive nuclei in each slide.

\section{RNA Isolation and RT-PCR Analysis}

Total RNAs from three frozen tissues (case nos. 1, 10, and 28) were obtained using RNeasy Mini kit (Qiagen, Tokyo, Japan) according to the manufacturer's instructions. Expression of NeuroD was examined by reverse transcription-polymerase chain reaction (RT-PCR) with the use of the following primers: forward primer, $5^{\prime}$-GTTATGAG ACTATCACTGCTCAGGACC-3'; reverse primer, 5'-AGAAGTTGCCATTGATGCTGAGC-3' 
Table 1 Clinicopathological features of the gastroenteropancreatic NET patients

\begin{tabular}{|c|c|c|c|c|c|c|}
\hline Case & Age/sex & Histology & Organ & Stage & Treatment & Prognosis (months) \\
\hline 1 & $58 / \mathrm{F}$ & NEC (large) & Rectum & III & Surgery+CT & NED (48) \\
\hline 2 & $56 / \mathrm{M}$ & NEC (small) & Rectum & IV & None & DOD (1) \\
\hline 3 & 69/M & NEC (small) & Rectum & III & Surgery+CT & DOD (3) \\
\hline 4 & $78 / \mathrm{M}$ & NEC (small) & Colon & IV & None & DOD (1) \\
\hline 5 & $47 / \mathrm{F}$ & NEC (large) & Colon & IV & Surgery+CT & DOD (6) \\
\hline 6 & $59 / \mathrm{F}$ & NEC (large) & Colon & IV & Surgery+CT & DOD (14) \\
\hline 7 & $81 / \mathrm{F}$ & NEC (small) & Stomach & IV & Surgery & DOD (7) \\
\hline 8 & $83 / \mathrm{F}$ & NEC (small) & Stomach & III & Surgery & DOD (5) \\
\hline 9 & $70 / \mathrm{M}$ & NEC (large) & Stomach & I & EMR & NED (30) \\
\hline 10 & $56 / \mathrm{M}$ & NEC (small) & Esophagus & IV & $\mathrm{CT}+\mathrm{RT}$ & DOD (4) \\
\hline 11 & $69 / \mathrm{F}$ & NEC (large) & Duodenum & IV & Surgery & NED (30) \\
\hline 12 & $58 / \mathrm{F}$ & NEC (small) & Gall bladder & IV & Surgery+CT & DOD (7) \\
\hline 13 & $19 / \mathrm{F}$ & NEC (small) & Pancreas & IV & Surgery & DOD (2) \\
\hline 14 & $60 / \mathrm{M}$ & NEC (large) & Pancreas & IV & CT & DOD (7) \\
\hline 15 & $59 / \mathrm{M}$ & NEC (well) & Pancreas & IV & Surgery+CT & NED (7) \\
\hline 16 & $69 / \mathrm{M}$ & NEC (well) & Pancreas & I & Surgery & NED (31) \\
\hline 17 & $67 / F$ & NEC (well) & Pancreas & I & Surgery & NED (23) \\
\hline 18 & $59 / \mathrm{F}$ & NEC (well) & Pancreas & I & Surgery & NED (71) \\
\hline 19 & $55 / \mathrm{F}$ & NEC (well) & Pancreas & I & Surgery & NED (65) \\
\hline 20 & $33 / \mathrm{F}$ & NEC (well) & Duodenum & IV & Surgery+TACE & AWD (57) \\
\hline 21 & $57 / \mathrm{M}$ & NEC (well) & Pancreas & I & Surgery & NED (5) \\
\hline 22 & $44 / F$ & NET (well) & Pancreas & I & Surgery & NED (53) \\
\hline 23 & $57 / \mathrm{F}$ & Carcinoid & Rectum & I & EMR & NED (39) \\
\hline 24 & $78 / \mathrm{M}$ & Carcinoid & Rectum & I & EMR & NED (32) \\
\hline 25 & $74 / \mathrm{M}$ & Carcinoid & Rectum & I & EMR & NED (36) \\
\hline 26 & $62 / F$ & Carcinoid & Rectum & I & EMR & NED (39) \\
\hline 27 & $46 / \mathrm{M}$ & Carcinoid & Rectum & I & EMR & NED (40) \\
\hline 28 & $76 / \mathrm{M}$ & Carcinoid & Rectum & I & EMR & NED (50) \\
\hline 29 & $61 / \mathrm{F}$ & Carcinoid & Rectum & I & EMR & NED (39) \\
\hline 30 & $50 / \mathrm{M}$ & Carcinoid & Duodenum & I & Surgery & NED (41) \\
\hline 31 & $44 / \mathrm{M}$ & Carcinoid & Duodenum & I & EMR & NED (34) \\
\hline 32 & $57 / \mathrm{F}$ & Carcinoid & Duodenum & I & Surgery & NED (32) \\
\hline 33 & $42 / \mathrm{M}$ & Carcinoid & Stomach & I & EMR & NED (81) \\
\hline
\end{tabular}

F, female; M, male; CT, chemotherapy; RT, radiation; EMR, endoscopic mucosal resection; TACE, transarterial chemoembolization; NED, no evidence of disease; AWD, alive with disease; DOD, dead of disease; NEC, neuroendocrine carcinoma; NET, neuroendocrine tumor.

For internal control, b-actin cDNA was amplified. The condition of PCR were initial denaturing at $95^{\circ} \mathrm{C}$ for $10 \mathrm{~min}$, followed by 35 cycles of denaturing at $94^{\circ} \mathrm{C}$ for $60 \mathrm{~s}$, annealing at $58^{\circ} \mathrm{C}$ for $60 \mathrm{~s}$, and extension at $72^{\circ} \mathrm{C}$ for $90 \mathrm{~s}$. All PCR products were visualized by electrophoresis and ethidium bromide staining in $2 \%$ agarose gels. RT-PCR was performed in a triplicate.

\section{Statistical Analysis}

All analyses were performed using SPSS 14.0 software (SPSS Inc., Chicago, IL, USA). Fisher's test was applied for comparisons. Survival data were analyzed according to the Kaplan-Meier method. Results were considered significant when $P<0.05$ was obtained.

\section{Results}

A total of 33 gastroenteropancreatic neuroendocrine tumor patients were included in the study. Clinicopathological features of the 33 patients are listed in Table 1.

\section{Expression of mASH1 in Gastroenteropancreatic Neuroendocrine Tumors}

Immunohistochemistry of hASH1 was done using anti-mASH1 monoclonal antibody. This antibody is known to cross-react to hASH1 and is reliable to detect hASH1 on human paraffin-embedded tissues. $^{4,7}$ In the present study the expression of mASH1 was found positive in 11 (33\%) of 33 gastroenteropancreatic neuroendocrine tumor patients. In the present study, nuclear staining was counted as mASH1 positive (Figure 1). The details of the immunohistochemical staining results are listed in Table 2. In regard to poorly differentiated neuroendocrine carcinomas, 10 (71\%) of 14 patients showed positive for mASH1. Only 1 (5\%) of 19 welldifferentiated neuroendocrine tumor and carcinoma patients showed positive for mASH1. mASH1 was significantly positive for poorly differentiated neuroendocrine carcinoma (Table 3a). Five (63\%) of eight small-cell carcinoma patients and five (83\%) of six large-cell carcinoma patients showed positive for mASH1, but there was no statistical significance between the histological phenotype (ie small cell and large cell; Table 3b). 


\section{Expression of NeuroD in Gastroenteropancreatic} Neuroendocrine Tumors

For the evaluation of NeuroD, - and + was considered low-expression group, ++ and +++
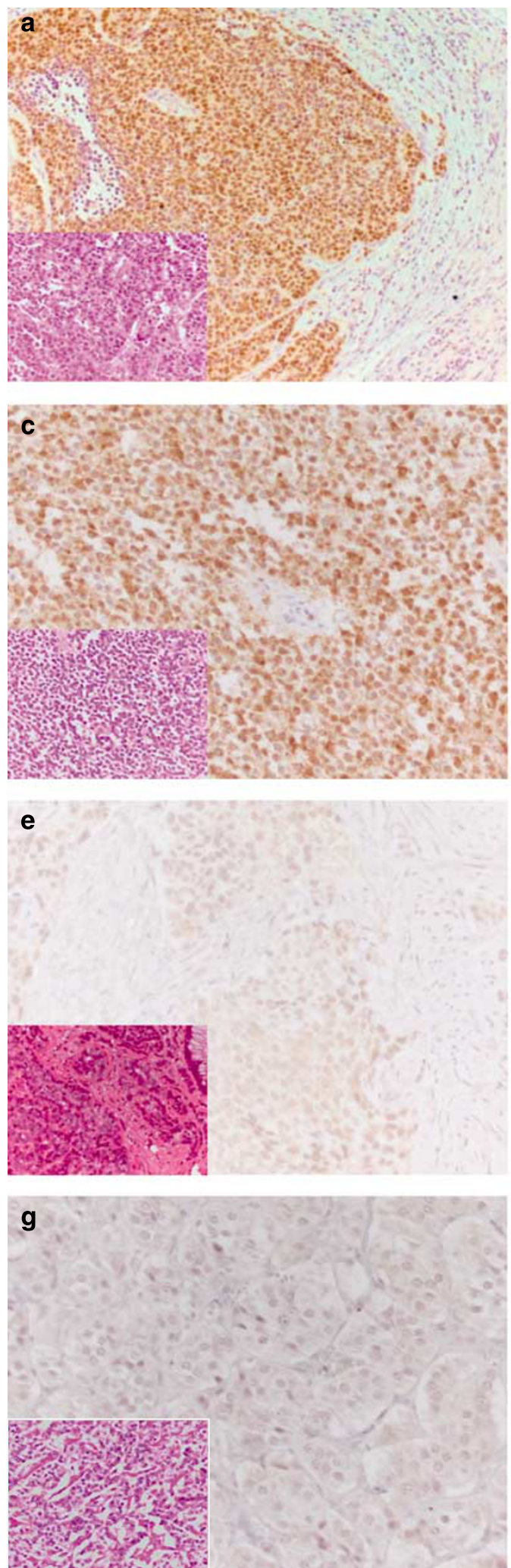

was considered high-expression group. In the present study $28(85 \%)$ of 33 gastroenteropancreatic neuroendocrine tumor patients showed high expression for NeuroD. NeuroD was considered to be positive when cytoplasmic or nuclear staining was
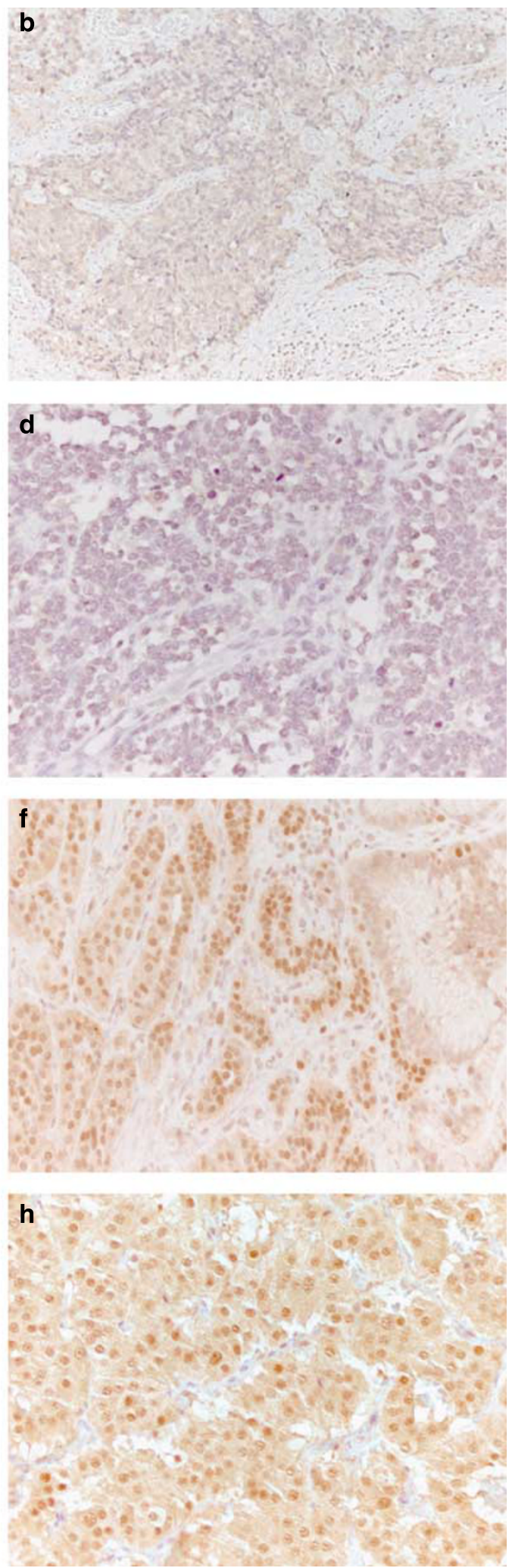
observed (Figure 1). The details of the immunohistochemical staining results are listed in Table 2. All the well-differentiated neuroendocrine tumor and carcinoma patients $(n=19)$ showed high expression to NeuroD. However with regard to poorly differentiated neuroendocrine carcinoma, $5(36 \%)$ of 14 patients showed low expression to NeuroD (Table 4a). For small-cell carcinoma, five (63\%) of eight patients showed low expression to NeuroD, whereas all six large-cell carcinoma patients showed high NeuroD expression (Table 4b). The expression pattern of NeuroD was statistically significant $(P=0.028)$ between the two histological phenotypes (ie small cell and large cell).

Table 2 Immunohistochemical results of gastroenteropancreatic NET

\begin{tabular}{|c|c|c|c|c|c|}
\hline Case & $m A S H 1$ & NeuroD & $\begin{array}{l}\text { Chromo- } \\
\text { graninA }\end{array}$ & $\begin{array}{l}\text { Synapto- } \\
\text { physin }\end{array}$ & $\begin{array}{c}K i-67 \\
(\%)\end{array}$ \\
\hline 1 & + & ++ & ++ & ++ & 18 \\
\hline 2 & ++ & - & - & - & 73 \\
\hline 3 & +++ & - & - & - & 82 \\
\hline 4 & - & ++ & - & ++ & 69 \\
\hline 5 & ++ & +++ & +++ & +++ & 73 \\
\hline 6 & + & ++ & - & ++ & 70 \\
\hline 7 & +++ & + & +++ & +++ & 70 \\
\hline 8 & - & ++ & - & +++ & 60 \\
\hline 9 & - & +++ & ++ & +++ & 60 \\
\hline 10 & +++ & - & - & - & 75 \\
\hline 11 & + & ++ & ++ & ++ & 65 \\
\hline 12 & - & +++ & ++ & +++ & 80 \\
\hline 13 & +++ & + & + & + & 40 \\
\hline 14 & ++ & +++ & - & - & 20 \\
\hline 15 & - & +++ & +++ & +++ & 10 \\
\hline 16 & - & ++ & +++ & ++ & 1 \\
\hline 17 & - & +++ & ++ & +++ & 2 \\
\hline 18 & - & +++ & ++ & +++ & 1 \\
\hline 19 & - & +++ & +++ & ++ & 1 \\
\hline 20 & - & ++ & ++ & +++ & 1 \\
\hline 21 & - & +++ & +++ & +++ & 1 \\
\hline 22 & + & ++ & +++ & +++ & 1 \\
\hline 23 & - & +++ & - & +++ & 0.16 \\
\hline 24 & - & +++ & - & +++ & 0.3 \\
\hline 25 & - & +++ & - & +++ & 0.7 \\
\hline 26 & - & +++ & ++ & +++ & 0.2 \\
\hline 27 & - & ++ & - & +++ & 0.8 \\
\hline 28 & - & +++ & ++ & +++ & 0.2 \\
\hline 29 & - & +++ & ++ & +++ & 0.3 \\
\hline 30 & - & ++ & +++ & ++ & 0.5 \\
\hline 31 & - & +++ & +++ & ++ & 0.2 \\
\hline 32 & - & +++ & + & +++ & 1 \\
\hline 33 & - & +++ & +++ & +++ & 1.7 \\
\hline
\end{tabular}

\section{NeuroD mRNA Expression by RT-PCR}

All poorly differentiated neuroendocrine carcinoma (both small cell and large cell) and welldifferentiated neuroendocrine tumor (carcinoid) showed NeuroD expression at RNA level (Figure 2). However, poorly differentiated neuroendocrine carcinoma (small-cell type) showed the lowest RNA expression (Figure 2).

\section{Survival Analysis}

There was a significant difference of overall survival between poorly differentiated neuroendocrine carcinoma and well-differentiated neuroendocrine carcinoma and tumor patients (log-rank test, $P<0.0001)$. The prognosis of poorly differentiated neuroendocrine carcinoma was extremely poor. With regard to poorly differentiated neuroendocrine carcinoma, there was no statistical significance of overall survival between mASH1-positive and -negative patients (Figure 3a). Moreover Ki67 positivity rate (cutoff rate of $60 \%$ ) did not show

Table 3a Correlation of mASH1 expression with WHO classifications

\begin{tabular}{lccc}
\hline WHO classification & mASH1(+) & mASH1(-) & Total \\
\hline $\begin{array}{l}\text { Well-differentiated } \\
\text { neuroendocrine tumor }\end{array}$ & 1 & 18 & 19 \\
$\quad \begin{array}{l}\text { and well-differentiated } \\
\text { neuroendocrine carcinoma }\end{array}$ & & & \\
$\begin{array}{l}\text { Poorly differentiated } \\
\quad \text { neuroendocrine carcinoma }\end{array}$ & 10 & 4 & 14 \\
Total & 11 & 22 & 33
\end{tabular}

WHO, World Health Organization.

$P=0.0001$ by Fisher's test.

Table 3b mASH1 expression in poorly differentiated neuroendocrine carcinoma

\begin{tabular}{lccc}
\hline & mASH1(+) & mASH1(-) & Total \\
\hline Small-cell carcinoma & 5 & 3 & 8 \\
Large-cell carcinoma & 5 & 1 & 6
\end{tabular}

WHO, World Health Organization.

$P=0.41$ by Fisher's test.

Figure 1 Immunohistochemical staining of mASH1 and NeuroD. (a) Positive staining of mASH1 in the poorly differentiated neuroendocrine carcinoma of the stomach (small-cell type; $\times 200$ inset). (a) H\&E staining of $\times 400$. (b) Positive staining of mASH1 in the poorly differentiated neuroendocrine carcinoma of pancreas (small-cell type; $\times 200$ inset). (b) H\&E staining of $\times 400$. (c) Negative-faint staining of mASH1 in the well-differentiated neuroendocrine tumor (carcinoid) of the rectum $(\times 400$ inset). (c) H\&E staining of $\times 400$. (d) Negative-faint staining of mASH1 in the well-differentiated neuroendocrine tumor of pancreas ( $\times 400$ inset). (d) H\&E staining of $\times 400$. (e) Low expression of NeuroD in the poorly differentiated neuroendocrine carcinoma of the stomach. Same case as (a, $\times 200)$. (f) Low expression of NeuroD in the poorly differentiated neuroendocrine carcinoma of pancreas. Same case as (b, $\times 400$ ). (g) High expression of NeuroD in the well-differentiated neuroendocrine tumor (carcinoid) of the rectum. Same case as $(\mathbf{c}, \times 400)(\mathbf{h})$ High expression of NeuroD in the well-differentiated neuroendocrine tumor of pancreas. Same case as $(\mathbf{d}, \times 400)$. 
Table 4a Correlation of NeuroD expression with WHO classifications

\begin{tabular}{lccc}
\hline WHO classification & $\begin{array}{c}\text { NeuroD } \\
\text { (high) }\end{array}$ & $\begin{array}{c}\text { NeuroD } \\
\text { (low) }\end{array}$ & Total \\
\hline $\begin{array}{l}\text { Well-differentiated } \\
\text { neuroendocrine tumor and } \\
\text { well-differentiated }\end{array}$ & 19 & 0 & 19 \\
$\begin{array}{c}\text { neuroendocrine carcinoma } \\
\text { Poorly differentiated } \\
\text { neuroendocrine carcinoma }\end{array}$ & 9 & 5 & 14 \\
\begin{tabular}{c} 
Total \\
\hline
\end{tabular} & 28 & 5 & 33 \\
\hline
\end{tabular}

WHO, World Health Organization.

$P=0.0084$ by Fisher's test.

Table 4b NeuroD expression in poorly differentiated neuroendocrine carcinoma

\begin{tabular}{lccc}
\hline & NeuroD (high) & NeuroD (low) & Total \\
\hline Small-cell carcinoma & 3 & 5 & 8 \\
Large-cell carcinoma & 6 & 0 & 6 \\
\hline
\end{tabular}

$P=0.028$ by Fisher's test.

statistical significance (Figure 3b). However, high NeuroD expression was associated with a better survival compared to low NeuroD expression in poorly differentiated neuroendocrine carcinoma patients (log-rank test, $P<0.05$; Figure 3c).

\section{Discussion}

Gastroenteropancreatic neuroendocrine tumors are rare diseases that present many clinical challenges. They secrete peptides and neuroamines that cause obvious clinical syndromes, including carcinoid syndrome. However, most gastroenteropancreatic neuroendocrine tumors are clinically silent until late presentation with mass effects. Management of these tumors should be highly individualized for each patient, considering the natural history of the tumor and general health of the patient. Surgery, radiological intervention, chemotherapy, and somatostatin analogues to control symptoms that result from released neuroamines and peptides are commonly used for treatment, but neither method has been reported to be fundamentally curative. The rarity, complexity, and heterogeneity of gastroenteropancreatic neuroendocrine tumors have contributed to the limitation of randomized trials and almost no survival increase has been admitted over the past 30 years. To establish a better treatment and improve the prognosis of gastroenteropancreatic neuroendocrine tumors, a better understanding of their biology is highly required, with emphasis on pathophysio-
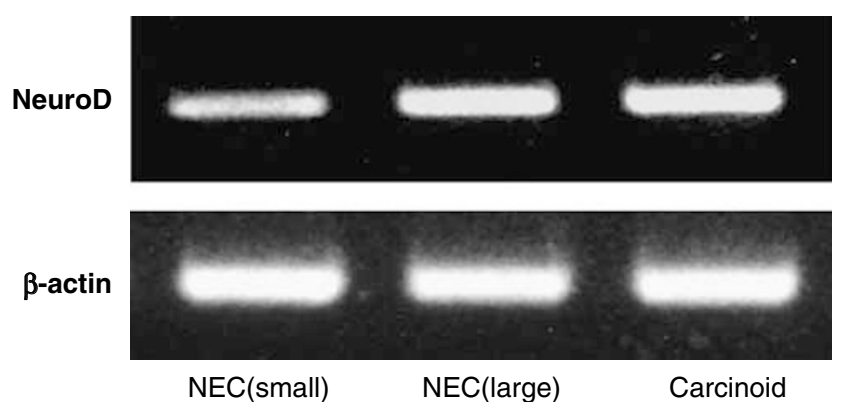

Figure 2 RT-PCR analysis of NeuroD by using fresh frozen samples. Poorly differentiated neuroendocrine carcinoma (smallcell type) was from esophagus (case no. 10), poorly differentiated neuroendocrine carcinoma (large-cell type) and carcinoid were from rectum (case nos. 1 and 28, respectively). Poorly differentiated neuroendocrine carcinoma (small-cell type) showed the lowest NeuroD expression.

logy at the molecular level (including transcription factors).

In the present study we focused on NeuroD and ASH1 both a member of the bHLH transcription factor that are essential for neural development. ${ }^{6,10,11}$ We previously reported that hASH1(mASH1) was aberrantly expressed in gastrointestinal neuroendocrine carcinomas but not in gastrointestinal carcinoids or adenocarcinomas. ${ }^{4}$ Also previous study of the lung has revealed that hASH1(mASH1) expression was dependent on histological differentiation, and was detected in less differentiated atypical carcinoids, as well as in large-cell neuroendocrine carcinomas and small-cell carcinomas, but was absent in fully differentiated typical carcinoids. $^{5}$

On the other hand, previous study revealed the positive expression of NeuroD in pulmonary large-cell neuroendocrine carcinoma and carcinoids but not in gastric small-cell carcinomas. $^{8,9}$ In the present study, mASH1 was highly (sensitivity of $71 \%$ ) and specifically (specificity of 95\%) expressed in poorly differentiated neuroendocrine carcinoma, which was in accordance with our previous study. However, positivity of mASH1 did not affect the prognosis of poorly differentiated neuroendocrine carcinoma patients. The prognostic significance of mASH1 expression in gastroenteropancreatic neuroendocrine tumors needs to be clarified in a larger number of patients.

In the present study, high NeuroD expression was seen in all gastroenteropancreatic welldifferentiated neuroendocrine carcinoma and tumor patients, however $36 \%$ (5 of 14 ) of the poorly differentiated neuroendocrine carcinoma patients showed low NeuroD expression. Surprisingly, the expression level of NeuroD had a significant impact on poorly differentiated neuroendocrine patients. Low NeuroD expression was associated with significant shorter overall survival. 
a

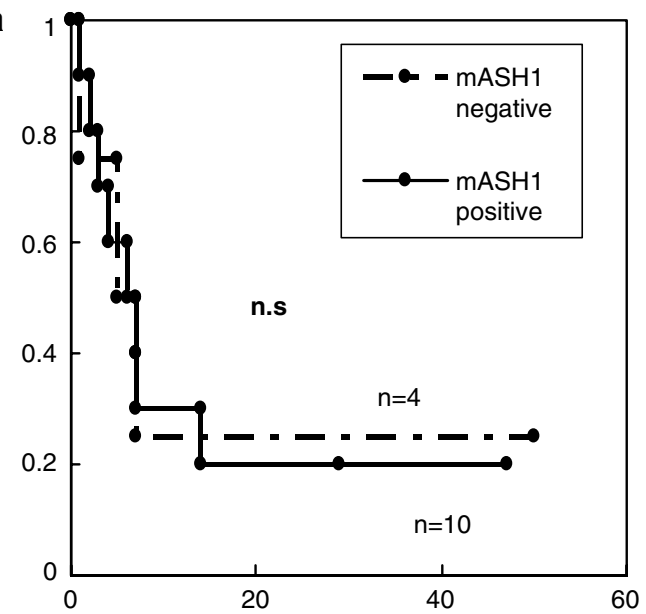

b

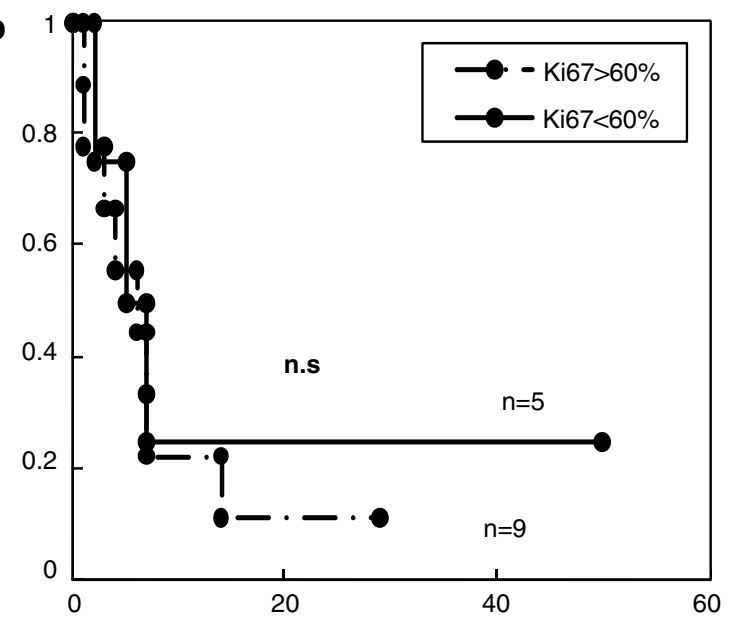

C

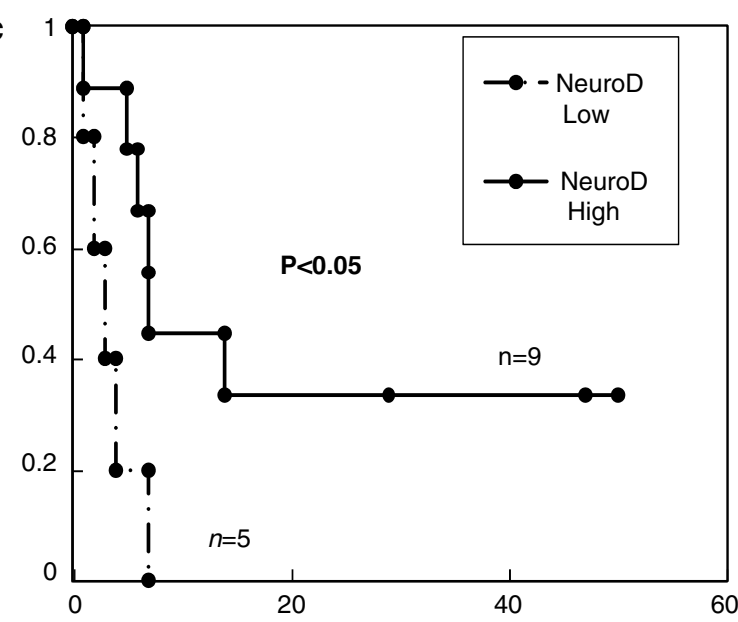

Figure 3 (a) Kaplan-Meier survival curves and log-rank test for poorly differentiated neuroendocrine carcinoma patients by mASH1 positivity. There was no statistical difference of overall survival between mASH1-positive and -negative patients. (b) Kaplan-Meier survival curves and log-rank test for poorly differentiated neuroendocrine carcinoma patients by Ki67 positivity. Ki67 (cutoff rate of $60 \%$ ) did not show statistical significance. (c) Kaplan-Meier survival curves and log-rank test for poorly differentiated neuroendocrine carcinoma patients by NeuroD expression. High NeuroD expression was associated with a better survival compared to low NeuroD expression $(P<0.05)$.
Poorly differentiated neuroendocrine carcinoma has been reported as highly malignant and can be divided into small- and large-cell neuroendocrine carcinomas. ${ }^{12}$ The prognostic significance between small-cell carcinoma and large cell carcinoma is still a matter of debate and morphological classification alone is not sufficient to differentiate neuroendocrine carcinomas with aggressive characteristics. ${ }^{13,14}$

The expression pattern of mASH1 and/or NeuroD might reflect the neuroendocrine differentiation of gastroenteropancreatic tumor. During neural development, mASH1 works at the initial phase transiently, and NeuroD works at the latter phase but is not expressed in nearby neuronal progenitors that are still undergoing cell division. ${ }^{6,15}$ These data together strongly suggest that mASH1 expression in gastroenteropancreatic neuroendocrine tumors mimic its early and transient expression pattern during development, and mASH1 may be necessary in the establishment of a cellular neuroendocrine phenotype, but may not be essential for the maintenance of the fully differentiated neuroendocrine phenotype. Moreover, NeuroD expression in gastroenteropancreatic neuroendocrine tumors may also imitate the neuronal differentiation because of its high positivity on well-differentiated neuroendocrine carcinomas and tumors in the present study. NeuroD may be essential for the maintenance of the fully differentiated neuroendocrine phenotype. The expression pattern of mASH1 and/or NeuroD in the present study may reflect the biological and pathophysiological difference of gastroenteropancreatic neuroendocrine tumors (especially between poorly differentiated neuroendocrine carcinoma and well-differentiated neuroendocrine tumor and carcinoma). This might be at least in part a clue to the worse prognosis for NeuroD-low poorly differentiated neuroendocrine carcinoma that may be a less differentiated tumor phenotype. In the present study, the tendency of the staining pattern of well-differentiated neuroendocrine tumor and carcinoma was mASH1 negative and NeuroD high, where as that of a poorly differentiated neuroendocrine carcinoma was mASH1 positive and NeuroD low.

Indeed mASH1-positive and NeuroD-low neuroendocrine carcinomas (five cases) showed the worst prognosis (MST for 3.4 months) among the gastroenteropancreatic neuroendocrine tumors. This type of tumor may be defined as 'undifferentiated neuroendocrine carcinoma' because three of five cases showed negative for conventional pan-neuroendocrine markers (chromograninA, synaptophysin).

Deeper insight into these neuronal transcription factors may surely be fruitful for the establishment of new criteria, prognostic factor, and treatment for gastroenteropancreatic neuroendocrine tumors. 


\section{References}

1 Barakat MT, Meeran K, Bloom SR. Neuroendocrine tumors. Endocr Relat Cancer 2004;11:1-18.

2 Kloppel G, Perren A, Heitz PU. The gastroenteropancreatic neuroendocrine cell system and its tumors: the WHO classification. Ann NY Acad Sci 2004;1014: 13-27.

3 Ball DW. Achaete-scute homologue-1 and Notch in lung neuroendocrine development and cancer. Cancer Lett 2004;204:159-169.

4 Shida T, Furuya M, Nikaido T, et al. Aberrant expression of human achate-scute homologue gene 1 in the gastrointestinal neuroendocrine carcinomas. Clin Cancer Res 2005;11:450-458.

5 Jiang SX, Kameya T, Asamura $\mathrm{H}$, et al. hASH1 expression is closely correlated with endocrine phenotype and differentiation extent in pulmonary neuroendocrine tumors. Mod Pathol 2004;17:222-229.

6 Lee JE, Hollenberg SM, Snider L, et al. Conversion of Xenopus ectoderm into neurons by NeuroD, a basic helix-loop-helix protein. Science 1995;268:836-844.

7 Oyama K, Sanno N, Teramoto A, et al. Expression of neuro D1 in human normal pituitaries and pituitary adenomas. Mod Pathol 2001;14:892-899.

8 Hiroshima K, Iyoda A, Shida T, et al. Distinction of pulmonary large cell neuroendocrine carcinoma from small cell lung carcinoma: a morphological, immunohistochemical, and molecular analysis. Mod Pathol 2006;19:1358-1368.
9 Fujii A, Kamiakito T, Takayashiki N, et al. Neuroendocrine tissue-specific transcription factor, BETA2/ NeuroD, in gastric carcinomas: a comparison with chromograninA and synaptophysin expressions. Pathol Res Pract 2003;199:513-519.

10 Borges M, Linnoila RI, van de Velde HJ, et al. An achaete-scute homologue essential for neuroendocrine differentiation in the lung. Nature 1997;386: 852-855.

11 Ito T, Udaka N, Yazawa T, et al. Basic helix-loop-helix transcription factors regulate the neuroendocrine differentiation of fetal mouse pulmonary epithelium. Development 2000;127:3913-3921.

12 Younossian AB, Brunbler MA, Totsch M. Feasibility of the new WHO classification of pulmonary neuroendocrine tumours. Swiss Med Wkly 2002;132: 535-540.

13 Matsui K, Jin XM, Kitagawa M, et al. Clinicopathological features of neuroendocrine carcinomas of the stomach: appraisal of small cell and large cell variants. Arch Pathol Lab Med 1998;122: 1010-1017.

14 Boo YJ, Park SS, Kim JH, et al. Gastric neuroendocrine carcinoma: clinicopathologic review and immunohistochemical study of E-cadherin and Ki-67 as prognostic markers. J Surg Oncol 2007;95: 110-117.

15 Cau E, Grafwohl G, Fode C, et al. Mash1 activates a cascade of bHLH regulators in olfactory neuron progenitors. Development 1997;124:1611-1621. 